\title{
查尔酮及其螺杂环衍生物的合成、晶体结构、抗氧化活性研究
}

\author{
$\begin{array}{ccccc}\text { 吴建章 } & \begin{array}{c}\text { 李物兰 } \\ \end{array} & \begin{array}{c}\text { 陈玲姿 } \\ \text { 卫 } \text { 涛 }^{b}\end{array} & \begin{array}{c}\text { 楚生辉 } \\ \text { 杨树林*,a }\end{array} & \text { 李校㫋 } \\ \end{array}$ \\ ( ${ }^{a}$ 南京理工大学生物工程研究所 南京 210094) \\ ( ${ }^{b}$ 温州医学院药学院 温州 325035) \\ (c温州医学院信息与工程学院 温州 325035)
}

\begin{abstract}
摘要 为了合成新结构类型查尔酮衍生物, 发现具有抗氧化活性的查尔酮类化合物, 设计合成了查尔酮 $\mathbf{A}$ 和螺杂环 $\mathbf{B}$ 两种类型, 共 21 个查尔酮类似物, 结构经 ESI-MS, ESI-HRMS 和 ${ }^{1} \mathrm{H}$ NMR 确认. 培养出螺杂环 $\mathbf{B} 1$ 的单晶, 通过 X 衍射 确证了其为单斜晶系. 其中螺杂环 $\mathbf{B}$ 为新结构类型化合物, 通过 1,3-偶极环加成反应，用不需加催化剂的 “一锅煮” 方 法合成，该反应具有很好的立体选择性和区域选择性、且环境友好. 用 DPPH 法测试了所有化合物的抗氧化活性，篮选 出了多个对 1,1-二苯基-2-三硝基苯肼(DPPH)自由基具有良好清除率的化合物, a 环 3,4-OH 取代的两类化合物都具有良 好的抗氧化活性，苯环邻位二羟基取代的查尔酮类化合物可能具有很好的抗氧化活性.
\end{abstract}

关键词 查尔酮; 螺杂环; 合成; DPPH; 抗氧化活性; 晶体结构

\section{Synthesis, Crystal Structure, Antioxidant Activity of Chalcones and Its Spiro-heterocyclic Analogues}

\author{
Wu, Jianzhang ${ }^{a, b}$ \\ Li, Wulan ${ }^{c}$ \\ Chen, Lingzi ${ }^{b}$ \\ Chu, Shenghui ${ }^{b}$ \\ Wei, Tao $^{b}$ \\ Yang, Shulin ${ }^{*, a}$ \\ Li, Xiaokun ${ }^{a, b}$ \\ Zhao, Chenguang $^{a}$ \\ $\left({ }^{a}\right.$ Institute of Biotechnology, Nanjing University of Science and Technology, Nanjing 210094) \\ $\left({ }^{b}\right.$ School of Pharmacy, Wenzhou Medical College, Wenzhou 325035) \\ ( ${ }^{c}$ College of Information Science and Computer Engineering, Wenzhou Medical College, Wenzhou 325035)
}

\begin{abstract}
To synthesize new structure type chalcone analogues, and obtain chalcone analogues with good antioxidant activity. Two types of chalcone analogs, chalcones $\mathbf{A}$ and spiro-heterocyclic $\mathbf{B}$ were designed and synthesized, and the structures of 21 compounds were characterized by ${ }^{1} \mathrm{H}$ NMR, ESI-MS and ESI-HRMS. Single-crystal of spiro-heterocyclic B was cultured, and its single-crystal structure was determined by X-ray diffraction study. The crystal structure of B1 was monoclinic system, space group $C 2 / c$, with cell dimensions of $a=21.350(3) \AA, b=8.6256(10) \AA, c=26.161(3) \AA$. Spiro-heterocyclic $\mathbf{B}$ is the new structure type, and obtained by one-pot synthesis which is 1,3-dipolar cycloaddition reaction and no catalyst. The synthesis of spiro-heterocyclic B is not only high regioselectivity and stereoselectivity, but also environmentally friendly. The antioxidant activities in vitro were evaluated by 1,1-diphenyl-2-picrylhydrazyl (DPPH) assay. Many compounds, which can have strong activity of scavenging DPPH free radicals, were screened. In the 2 types of chalcones analogs, the compounds with 3,4-(OH) in "a" ring have excellent antioxidant activities. The chalcone analogs with $o$-dihydroxy in benzene ring maybe have good antioxidant activities.
\end{abstract}

Keywords chalcones; spiro-heterocyclic; synthesis; DPPH; antioxidant activities; crystal structure

黄酮类化合物广泛存在于水果蔬菜和各种天然药 物中, 具有较好的抗氧化、抗肿瘤、抗炎、抗血管增生、
抗菌和抗㾏疾等生物活性 ${ }^{[1 \sim 3]}$. 查尔酮类化合物属于黄 酮类化合物, 近年来发现合成的查尔酮类化合物也具有

\footnotetext{
*E-mail: wjzwzmc@126.com,wujianzhang6@163.com

Received April 1, 2012; revised June 18, 2012; published online July 2, 2012.

Project supported by the Technology Foundation for Medical Science of Zhejiang Province (No. 2012KYA129), the Project of Wenzhou Sci \& Tech Bureau (No. 20090101), and the Technology Foundation for Chinese Medicine of Zhejiang Province (No. 2012ZB102).

浙江省医药卫生科技计划(No. 2012KYA129)、温州市科技局(No. Y20090101)、浙江省中医药科技计划 B 类(No. 2012ZB102)资助项目.
} 
很好的生物学活性，且该类化合物的毒性非常低，因此 吸引了越来越多科学家的目光 ${ }^{[4 \sim 6]}$. 到目前为止, 仅有 较少的文献报道了天然查尔酮的抗氧化活性, 而对合成 的查尔酮类化合物的抗氧化活性研究报道极少 ${ }^{[1,7 \sim 9]}$. 为了发掘抗氧化活性好的查尔酮类似物, 本文合成了经 典查尔酮(查尔酮 A)和螺杂环类查尔酮(螺杂环 B)两种 类型的查尔䣶类似物, 其中螺杂环 $\mathbf{B}$ 为新结构类型, 通 过单晶 X 衍射表征了螺杂环 $\mathbf{B}$ 的结构(Scheme 1). 采用 1,1-二苯基-2-三硝基苯肼(DPPH)法测试了化合物的体 外抗氧化活性, 发现了多个抗氧化活性较好的化合物.

\section{1 结果与讨论}

\section{1 合成}

查尔酩 $\mathbf{A}$ 类化合物的合成为常见的差醛缩合反应, 根据原料分子苯环上是否有羟基选择用碱性或酸性条 件进行合成, 都可以得到较高产率的产物. 但原料一般 都反应不完全, 所以沉淀析出的产物都需要柱层析或者 重结晶进行纯化. 螺杂环 $\mathbf{B}$ 为尚未有文献报道的新结构 类型的查尔酮类似物, 我们参考相应的螺杂环类单羰基

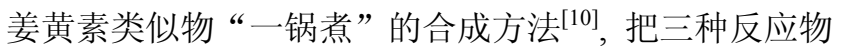
直接混合, 不加催化剂直接加热回流, 一般 $5 \mathrm{~h}$ 左右即 可完成反应，合成螺杂环 $\mathbf{B}$ 符合绿色合成化学的宗旨.

\subsection{B1 的晶体结构}

图 1 是化合物 B1 (CCDC 号为 829400)的分子晶体 结构图和晶胞堆积图. 晶体的分子式是 $\mathrm{C}_{27} \mathrm{H}_{28} \mathrm{~N}_{2} \mathrm{O}_{7}$, 分 子量 492.51 , 属于单斜晶系, 空间群为 $C 2 / c$, 晶胞参数 $a=21.350(3) \AA, b=8.6256(10) \AA, c=26.161(3) \AA$. 查尔 酮 $\mathrm{A}$ 类化合物骨架为经典查尔酮分子骨架, 整个分子是
高度共轭的结构，因此分子中两个苯环( “ $a$ ” 和 “ $b$ ” 环) 一般是近乎平面的结构. 查尔酮 $\mathbf{A}$ 类化合物反应形成螺 杂环 $\mathbf{B}$ 后, 共轭链在与偶极子反应的两个碳原子处中 断, 整个分子的由近乎平面结构变成了螺杂环立体结 构. B1 中来源于 $\mathbf{A 1}$ 的两个苯环( “ $\mathrm{a}$ ” 和 “ $\mathrm{b}$ ” 环)接近垂 直, 其二面角为 $80.07^{\circ}$, “ $\mathrm{a}$ ” 环与吲哚酮环平面二面角 为 $48.38^{\circ}$, “ $\mathrm{b}$ ” 环与吲哚酮环平面二面角为 $34.95^{\circ}$. “ $a$ ” 和 “ $b$ ” 环连接碳上存在 2 个手性碳, 绝对构型分别为 $2 R$ 和 $3 S$. “ “ $\mathrm{a}$ ”和 “ $\mathrm{b}$ ” 环之间连接羰基与吲哚酮羰基是 反式关系.

\section{3 螺杂环类查尔酮的反应机制及立体化学}

Kumar 等 ${ }^{[10,21]}$ 进行过类似的姜黄素螺杂环化合物的 合成研究，该类反应为 1,3-偶极环加成反应. 本文的螺 杂环 $\mathrm{B}$ 的合成也为此类反应. 反应的机制可能为，肌氨 酸与靛红亲核加成, 脱去二氧化碳, 生成以 $\mathrm{N}$ 原子为中 心的 1,3-偶极子(图 2). 由本文合成的螺杂环 $\mathbf{B}$ 的结构和 晶体结构可知，1,3-偶极子与查尔酮 $\mathbf{A}$ 加成时具有很好 的区域选择性和立体选择性: 偶极子 1 '和 3 '碳原子分别 加成到查尔酮 3 和 2 碳原子上, 未见偶极子 1 '和 3 '碳原 子分别加成到查尔酮 2 和 3 碳原子上的产物, 即表现出 区域选择性; 偶极子经历过渡态 “ $a$ ”, 得到螺杂环 $\mathbf{B}$ 产 物, 未见由过渡态 “ $b$ ” 所得的产物, 即表现出极好的立 体选择性.

\section{4 抗氧化活性}

查尔酮衍生物的对 DPPH 自由基的清除率结果见图 3 , 多个化合物都表现出了较好的活性. 化合物浓度为 $20 \mathrm{mg} / \mathrm{L}$ 时, 有 9 个化合物对 DPPH 自由基的清除率达

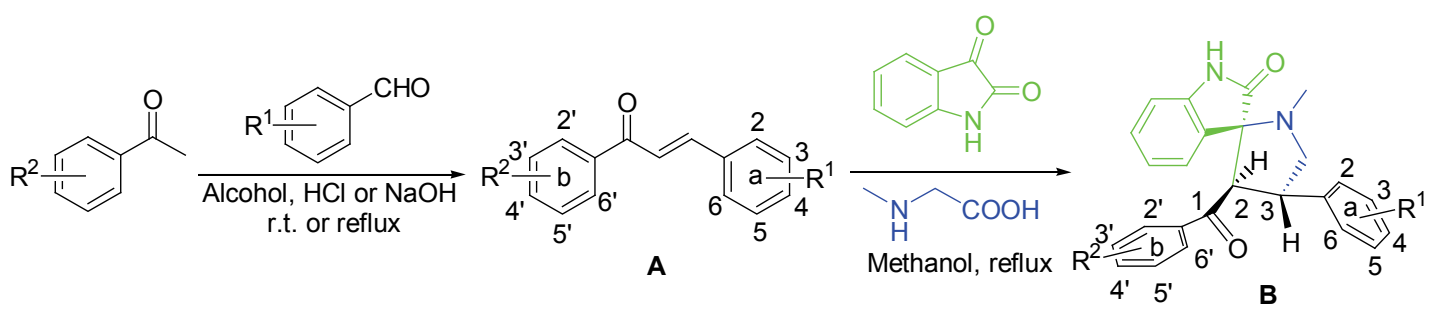

\begin{tabular}{|c|c|c|c|c|c|}
\hline Compd. & $\mathrm{R}^{1}$ & $\mathrm{R}^{2}$ & Compd. & $\mathrm{R}^{1}$ & $\mathrm{R}^{2}$ \\
\hline A1 & $3,4-(\mathrm{OH})_{2}$ & $2^{\prime}, 4^{\prime}-\left(\mathrm{OCH}_{3}\right)_{2}$ & A12 & $3,4-\left(\mathrm{OCH}_{3}\right)_{2}$ & 3'-OH \\
\hline A2 & $3,4-(\mathrm{OH})_{2}$ & $2^{\prime}-\mathrm{Cl}$ & A13 & $4-\mathrm{OCH}_{3}$ & 4'-OH \\
\hline A3 & $3,4-(\mathrm{OH})_{2}$ & $2^{\prime}, 4^{\prime}, 66^{\prime}-\left(\mathrm{OCH}_{3}\right)_{3}$ & A14 & $3,4,5-\left(\mathrm{OCH}_{3}\right)_{3}$ & 4'-OH \\
\hline A4 & $3,4-(\mathrm{OH})_{2}$ & $3^{\prime}, 4^{\prime}-\left(\mathrm{OCH}_{3}\right)_{2}$ & A15 & $4-\mathrm{OH}$ & 4'-OH \\
\hline A5 & $3,4-(\mathrm{OH})_{2}$ & 4'- $-\mathrm{OCH}_{3}$ & A16 & $4-\mathrm{OH}$ & $2^{\prime}, 4^{\prime}-\left(\mathrm{OCH}_{3}\right)_{2}$ \\
\hline A6 & $3,4-(\mathrm{OH})_{2}$ & 4'- $-\mathrm{OCH}_{2} \mathrm{CH}_{3}$ & A17 & $3-\mathrm{OH}$ & 3'-OH \\
\hline A7 & $3,4-\left(\mathrm{OCH}_{3}\right)_{2}$ & $2^{\prime}-\mathrm{Cl}$ & B1 & $3,4-(\mathrm{OH})_{2}$ & $2^{\prime}, 4^{\prime}-\left(\mathrm{OCH}_{3}\right)_{2}$ \\
\hline A8 & $3,4-\left(\mathrm{OCH}_{3}\right)_{2}$ & $2^{\prime}, 4^{\prime}, 66^{\prime}-\left(\mathrm{OCH}_{3}\right)_{3}$ & B2 & $3,4-(\mathrm{OH})_{2}$ & $2^{\prime}-\mathrm{Cl}$ \\
\hline A9 & $3,4-\left(\mathrm{OCH}_{3}\right)_{2}$ & $3^{\prime}, 4^{\prime}-\left(\mathrm{OCH}_{3}\right)_{2}$ & B3 & $3,4-\left(\mathrm{OCH}_{3}\right)_{2}$ & 2'-Cl \\
\hline $\mathbf{A 1 0}$ & $3,4-\left(\mathrm{OCH}_{3}\right)_{2}$ & 4'- $-\mathrm{OCH}_{3}$ & B4 & $3,4-(\mathrm{OH})_{2}$ & 4'- $-\mathrm{OCH}_{2} \mathrm{CH}_{3}$ \\
\hline
\end{tabular}



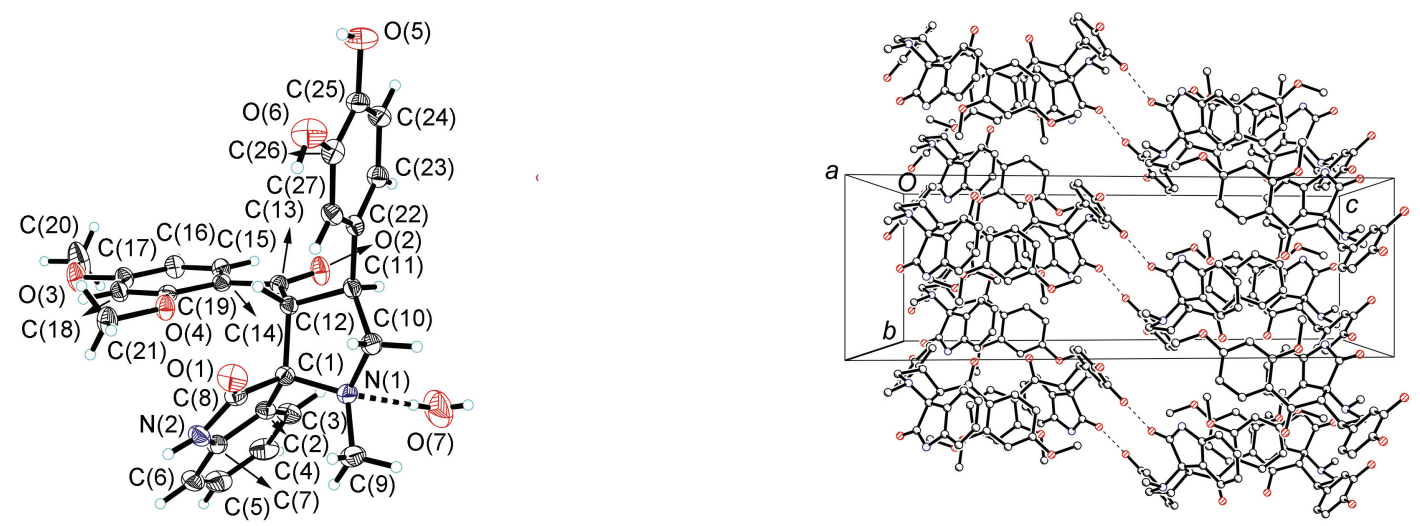

图 1 化合物 B1 的晶体结构和晶胞堆积图

Figure 1 Crystal structure and packing diagram of the unit cell of B1 Dotted lines indicate the hydrogen bonds<smiles>[R]c1ccc(C=CC(=O)c2ccc([R])cc2)cc1</smiles>

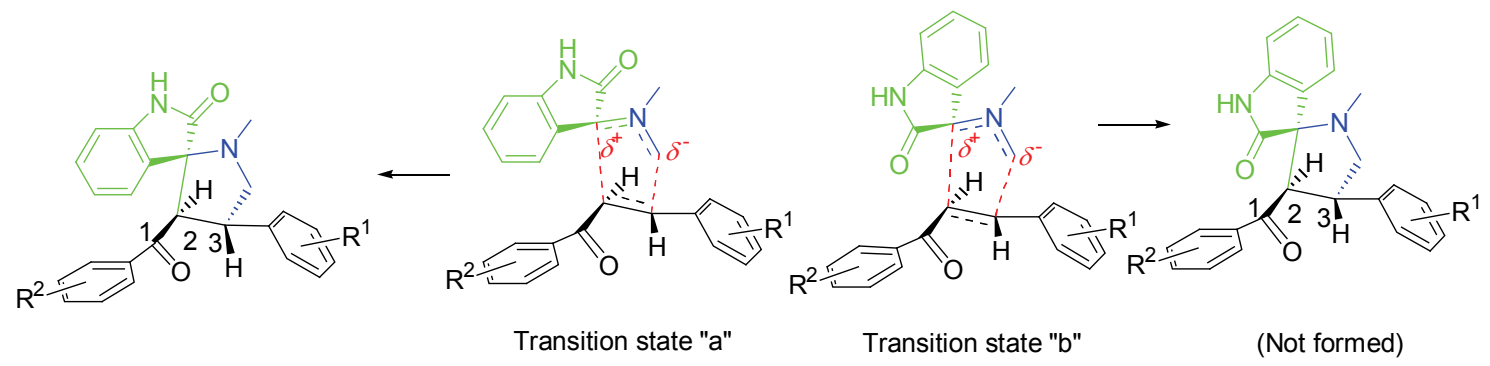

Production (formed)

图 2 合成螺杂环查尔酮的立体化学和可能的反应机制

Figure 2 Stereochemistry and possible reaction mechanism of synthesizing spiro-heterocyclic chalcones analogs

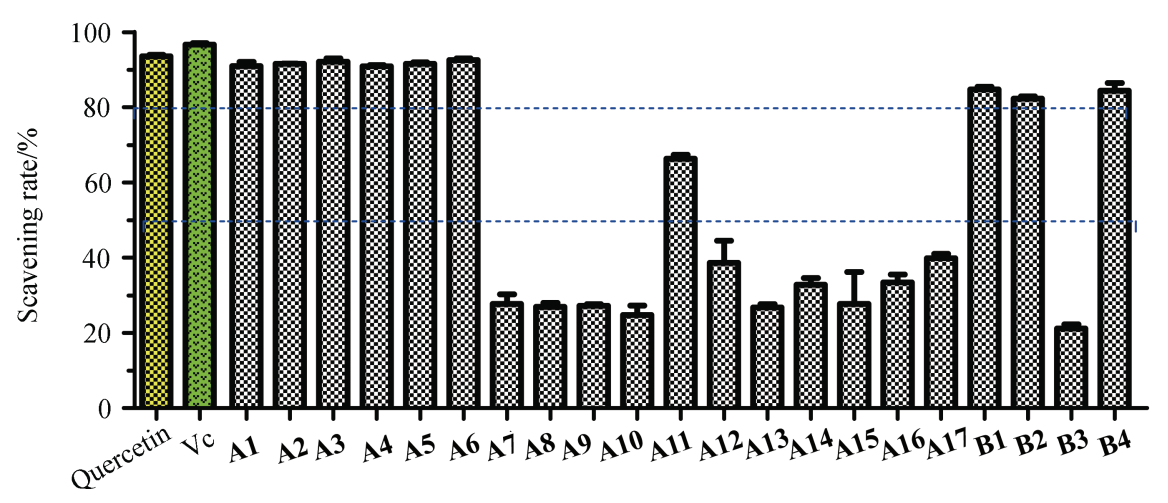

图 3 化合物对 DPPH 自由基的清除效果

Figure 3 Scavenging activity of compounds on DPPH free radical

到 $80 \%$ 以上，其活性接近阳性对照槲皮素(Quercetin)和 维生素 $\mathrm{C}(\mathrm{Vc})$.

构效关系分析显示, $\mathrm{a}$ 环上 3 和 4 位同时为着基的化 合物 $(\mathrm{A1} \sim \mathrm{A6}, \mathrm{B1}, \mathrm{B2}, \mathrm{B} 4)$, 都具有很好的抑制 DPPH 的
活性，在 $20 \mathrm{mg} / \mathrm{L}$ 时对 DPPH 的抑制率均达 $80 \%$ 以上; 苯环无差基取代的化合物(A7 A9, A11, B3)活性都很 差; 苯环单羟基取代化合物, 如果羟基邻位无甲氧基时 (A12 A17)活性也很差; 而羟基邻位有甲氧基的单羟 
基取代化合物(A11)，具有较好的活性，在 $20 \mathrm{mg} / \mathrm{L}$ 时对 DPPH 的抑制率达 $65 \%$.

对 DPPH 的抑制活性, 主要与化合物的酚羟基脱氢 的难易有关. 3,4-OH 活性较好的原因可能为, 两个羟基 处于邻位时，一个羟基的氢原子与另一个羟基的氧原子 容易形成分子内氢键; 分子内氢键的形成使其去氢后的 自由基更加稳定, 表明该类型衍生物更容易发生抽氢反 应从而发挥较为理想的抗氧化活性. 而邻位有甲氧基的 单羟基取代化合物 $\mathbf{A 1 0}$, 其 4-OH 也会与 $3-\mathrm{OCH}_{3}$ 形成分 子内氢键, 同理导致化合物具有较好的抗氧化活性. 邻 位无甲氧基的单羟基取代化合物的羟基不能形成分子 内氢键，所以其活性与无羟基化合物类似.

\section{2 结论}

设计合成出新型的螺杂环类查尔酮衍生物, 该类化 合物的合成具有一步合成、绿色环保、高度的立体和区 域选择性等优点, 为查尔酮类似物的结构研究提供了新 的实验和理论依据.

发现了多个对 DPPH 具有良好抑制活性的查尔酮类 似物; 3,4-OH 为邻位着基取代, 推测其它相邻二羟基取 代查尔酮类似物由于分子内氢键的形成, 都会具有良好 的清除 DPPH 的活性; 为新型查尔酮类抗氧化剂的研究 提供了实验参考.

\section{3 实验部分}

\section{1 仪器与试剂}

Fisher-Johns 型显微熔点仪, Bruker AVANCE III $600 \mathrm{MHz}$ 超导核磁共振仪, Bruker Esquire 3000 质谱仪, Bruker micrOTOF-Q II 质谱仪, Bruker SMARTAPEX II-CCDX-射线单晶衍射仪, Anthos Zenyth200rt 酶标仪. $\mathrm{DPPH}$, 䚞皮素(BR)与抗坏血酸(AR)购于国药集团化学 试剂有限公司, 其它所用试剂均为分析纯. 薄层和柱层 析用硅胶均为青岛海洋化工厂产品.

\section{2 查尔酮类似物的设计与合成}

设计了经典查尔酮(查尔酮 $\mathbf{A})$ 和螺杂环类查尔酮 (螺杂环 B)两种结构类型共 22 个化合物. 化合物苯环上 的酚羟基一般与其抗氧化活性具有相关性, 因此所设计 化合物大部分含有酚羟基. 查尔酮 $\mathbf{A}$ 类化合物的合成为 简单的羟醛缩合反应, 螺杂环 $\mathbf{B}$ 的合成为 1,3-偶极环加 成反应。

\subsection{1 查尔酮 $\mathbf{A}$ 的合成}

将 $1 \mathrm{mmol}$ 取代苯甲醛溶解在 $10 \mathrm{~mL}$ 左右无水乙醇 中, 再加入等物质的量的取代苯乙酮. 如果取代苯甲醛 或取代苯乙酮的取代基无羟基，滴加 5 滴左右的 $40 \%$
$\mathrm{NaOH}$ 作为催化剂, 反应温度控制在 $4{ }^{\circ} \mathrm{C}$ 左右; 取代基 如果有羟基, 通入 $\mathrm{HCl}$ 气体作为催化剂, 常温下如不反 应, 将逐步升高温度, 直至反应. 用 TLC 监测反应的进 行, 反应完后, 反应液中加入 $1 \sim 3$ 倍于反应液体积的 水, $4{ }^{\circ} \mathrm{C}$ 静置 $0.5 \sim 10 \mathrm{~h}$ 左右后有沉淀析出, 过滤, 用 $5 \% \sim 10 \%$ 的乙醇水溶液洗涤沉淀, 真空干燥后, 用硅胶 柱层析纯化(洗脱溶剂为石油醚: 乙酸乙酯或者氯仿: 甲醇的混合溶剂)得到各种查尔酮 $\mathbf{A}$ 类化合物.

(E)-3-(3,4-二羟基苯基)-1-(2,4-二甲氧基苯基)丙-2烯-1-酮(A1): 黄绿色粉末, 产率 81.55\%. m.p. 151.7 $155.3{ }^{\circ} \mathrm{C}$ (Lit. $\left.{ }^{[11]} 160 \sim 161{ }^{\circ} \mathrm{C}\right) ;{ }^{1} \mathrm{H}$ NMR $\left(\mathrm{CD}_{3} \mathrm{COCD}_{3}\right) \delta$ : 7.659 (d, $\left.J=8.4 \mathrm{~Hz}, 1 \mathrm{H}, \mathrm{H}-6^{\prime}\right), 7.500$ (d, $J=15.6 \mathrm{~Hz}, 1 \mathrm{H}$, $\mathrm{H}-\beta), 7.399$ (d, $J=15.6 \mathrm{~Hz}, 1 \mathrm{H}, \mathrm{H}-\alpha), 7.221$ (d, $J=2.4 \mathrm{~Hz}$, 1H, H-2), 7.059 (dd, $J=1.8,8.4 \mathrm{~Hz}, 1 \mathrm{H}, \mathrm{H}-6), 6.878$ (d, $J=7.8 \mathrm{~Hz}, 1 \mathrm{H}, \mathrm{H}-5$ ), 6.673 (d, $\left.J=2.4 \mathrm{~Hz}, 1 \mathrm{H}, \mathrm{H}-3^{\prime}\right), 6.632$ (dd, $\left.J=2.4,8.4 \mathrm{~Hz}, 1 \mathrm{H}, \mathrm{H}-5^{\prime}\right), 3.956$ (s, $3 \mathrm{H}, \mathrm{OCH}_{3}-2^{\prime}$ ), 3.894 (s, 3H, $\left.\mathrm{OCH}_{3}-4^{\prime}\right)$; ESI-MS $m / z$ : $301.1(\mathrm{M}+\mathrm{H})^{+}$.

(E)-1-(2-氯苯基)-3-(3,4-二羟基苯基)丙-2-烯-1-酮 (A2): 棕黄色粉末, 产率 $81.61 \%$. m.p. $144.3 \sim 145.7{ }^{\circ} \mathrm{C}$ (Lit. ${ }^{[1]} 174 \sim 178{ }^{\circ} \mathrm{C}$ ); ${ }^{1} \mathrm{H}$ NMR (DMSO- $d_{6}$ ) $\delta: 8.319$ (brs, 2H, OH-3, OH-4), $7.500 \sim 7.538$ (m, 3H, H-3', H-4', H-6'), $7.451 \sim 7.486\left(\mathrm{~m}, 1 \mathrm{H}, \mathrm{H}-5^{\prime}\right), 7.315(\mathrm{~d}, J=16.2 \mathrm{~Hz}, 1 \mathrm{H}$, H- $\beta$ ), 7.226 (d, $J=2.4 \mathrm{~Hz}, 1 \mathrm{H}, \mathrm{H}-2), 7.090$ (d, $J=1.8 \mathrm{~Hz}$, $8.4 \mathrm{~Hz}, 1 \mathrm{H}, \mathrm{H}-6), 6.955$ (d, $J=16.2 \mathrm{~Hz}, 1 \mathrm{H}, \mathrm{H}-\alpha), 6.988$ (d, $J=8.4 \mathrm{~Hz}, 1 \mathrm{H}, \mathrm{H}-5$ ); ESI-MS $m / z: 275.2[\mathrm{M}+\mathrm{H}]^{+}$.

(E)-3-(3,4-二羟基苯基)-1-(2,4,6-三甲氧基苯基)丙2-烯-1-酮(A3): 黄绿色稠状物, 产率 78.6\%. ${ }^{1} \mathrm{H}$ NMR $\left(\mathrm{CDCl}_{3}\right) \delta: 7.231$ (d, $\left.J=15.6 \mathrm{~Hz}, 1 \mathrm{H}, \mathrm{H}-\beta\right), 7.039$ (d, $J=$ $1.8 \mathrm{~Hz}, 1 \mathrm{H}, \mathrm{H}-2), 6.910$ (dd, $J=1.8,8.4 \mathrm{~Hz}, 1 \mathrm{H}, \mathrm{H}-6$ ), $6.816(\mathrm{~d}, J=8.4 \mathrm{~Hz}, 1 \mathrm{H}, \mathrm{H}-5), 6.760$ (d, $J=15.6 \mathrm{~Hz}, 1 \mathrm{H}$, $\mathrm{H}-\alpha$ ), 6.187 (s, 2H, H-3', H-5'), 3.757 (s, 6H, OCH -2 ', $\left.\mathrm{OCH}_{3}-6^{\prime}\right), 3.681$ (s, $\left.3 \mathrm{H}, \mathrm{OCH}_{3}-4^{\prime}\right)$; ESI-MS $m / z: 331.4$ $[\mathrm{M}+\mathrm{H}]^{+}$; HRMS calcd for $\mathrm{C}_{18} \mathrm{H}_{18} \mathrm{O}_{6}[\mathrm{M}+\mathrm{H}]^{+}$331.1103, found 331.1158 .

(E)-3-(3,4-二羟基苯基)-1-(3,4-二甲氧基苯基)丙-2烯-1-酮(A4): 黄色粉末, 产率 58.5\%. m.p. 88.3 $93.7{ }^{\circ} \mathrm{C}$ (Lit. $\left.{ }^{[11]} 132 \sim 137{ }^{\circ} \mathrm{C}\right) ;{ }^{1} \mathrm{H}$ NMR $\left(\mathrm{CD}_{3} \mathrm{COCD}_{3}\right) \delta: 7.838$ (dd, $\left.J=1.8,8.4 \mathrm{~Hz}, 1 \mathrm{H}, \mathrm{H}-6^{\prime}\right), 7.670$ (d, $J=15.6 \mathrm{~Hz}, 1 \mathrm{H}$, H- $\beta$ ), 7.663 (d, $\left.J=1.8 \mathrm{~Hz}, 1 \mathrm{H}, \mathrm{H}-2^{\prime}\right), 7.629$ (d, $J=15.6$ $\mathrm{Hz}, 1 \mathrm{H}, \mathrm{H}-\alpha), 7.317$ (d, $J=1.8 \mathrm{~Hz}, 1 \mathrm{H}, \mathrm{H}-2), 7.170$ (dd, $J=1.8,8.4 \mathrm{~Hz}, 1 \mathrm{H}, \mathrm{H}-6), 7.089$ (d, $J=8.4 \mathrm{~Hz}, 1 \mathrm{H}, \mathrm{H}-5^{\prime}$ ), 7.901 (d, $J=8.4 \mathrm{~Hz}, 1 \mathrm{H}, \mathrm{H}-5), 3.892$ (s, 6H, $\mathrm{OCH}_{3}-3$ ', $\left.\mathrm{OCH}_{3}-4^{\prime}\right)$; ESI-MS $m / z$ : $301.2[\mathrm{M}+\mathrm{H}]^{+}$.

(E)-3-(3,4-二羟基苯基)-1-(4-甲氧基苯基)丙-2-烯-1酮(A5): 红棕色粉末, 产率 $45.5 \%$. m.p. $170.4 \sim 172.9{ }^{\circ} \mathrm{C}$ 
(Lit. $\left.{ }^{[12]} 172 \sim 178{ }^{\circ} \mathrm{C}\right)$.

(E)-3-(3,4-二甲氧基苯基)-1-(4-乙氧基苯基)丙-2烯-1-酮(A6): 黄色粉末, 产率 66.51\%. m.p. 168.7 $170.3{ }^{\circ} \mathrm{C}$ (Lit. $\left.{ }^{[11]} 172 \sim 178{ }^{\circ} \mathrm{C}\right)$.

(E)-1-(2-氯苯基)-3-(3,4-二甲氧基苯基)丙-2-烯-1-酮 (A7): 淡黄色粉末, 产率 92.38\%. m.p. 93.2 96.0 ${ }^{\circ} \mathrm{C} ;{ }^{1} \mathrm{H}$ NMR $\left(\mathrm{CDCl}_{3}\right) \delta: 7.452 \sim 7.468(\mathrm{~m}, 2 \mathrm{H}, \mathrm{H}-3$ ', H-6'), 7.416 (dt, $J=1.8 \mathrm{~Hz}, 7.8 \mathrm{~Hz}, 1 \mathrm{H}, \mathrm{H}-4$ '), 7.382 (d, $J=16.2 \mathrm{~Hz}$, $1 \mathrm{H}, \mathrm{H}-\beta), 7.364$ (dt, $\left.J=1.2,7.8 \mathrm{~Hz}, 1 \mathrm{H}, \mathrm{H}-5^{\prime}\right), 7.142$ (dd, $J=1.8,8.4 \mathrm{~Hz}, 1 \mathrm{H}, \mathrm{H}-6), 7.088$ (d, $J=2.4 \mathrm{~Hz}, 1 \mathrm{H}, \mathrm{H}-2)$, $6.991(\mathrm{~d}, J=16.2 \mathrm{~Hz}, 1 \mathrm{H}, \mathrm{H}-\alpha), 6.878$ (d, $J=8.4 \mathrm{~Hz}, 1 \mathrm{H}$, $\mathrm{H}-5), 3.951$ (s, 6H, $\left.\mathrm{OCH}_{3}-3, \mathrm{OCH}_{3}-4\right)$; ESI-MS m/z: 303.3 $[\mathrm{M}+\mathrm{H}]^{+} ;$HRMS calcd for $\mathrm{C}_{17} \mathrm{H}_{15} \mathrm{ClO}_{3}[\mathrm{M}+\mathrm{H}]^{+}$ 303.0710, found 303.0782.

(E)-3-(3,4-二甲氧基苯基)-1-(2,4,6-三甲氧基苯基) 丙-2-烯-1-酮(A8): 黄色粉末，产率 62.73\%. m.p. 104 $106{ }^{\circ} \mathrm{C} ;{ }^{1} \mathrm{H} \mathrm{NMR}\left(\mathrm{CDCl}_{3}\right) \delta: 7.282(\mathrm{~d}, J=16.2 \mathrm{~Hz}, 1 \mathrm{H}$, H- $\beta$ ), 7.088 (d, $J=8.4 \mathrm{~Hz}, 1 \mathrm{H}, \mathrm{H}-6), 7.065$ (s, 1H, H-2), 6.849 (d, $J=8.4 \mathrm{~Hz}, 1 \mathrm{H}, \mathrm{H}-5), 6.843$ (d, $J=15.6 \mathrm{~Hz}, 1 \mathrm{H}$, H- $\alpha$ ), 6.169 (s, 2H, H-3', H-5'), 3.902 (s, 6H, $\mathrm{OCH}_{3}-2^{\prime}$, $\mathrm{OCH}_{3}-6$ ), 3.863 (s, 3H, $\mathrm{OCH}_{3}-3$ ), 3.771 (s, 6H, $\mathrm{OCH}_{3}-4^{\prime}$, $\left.\mathrm{OCH}_{3}-4\right)$; ESI-MS m/z: $358.9[\mathrm{M}+\mathrm{H}]^{+}$; HRMS calcd for $\mathrm{C}_{20} \mathrm{H}_{22} \mathrm{O}_{6}[\mathrm{M}+\mathrm{H}]^{+}$359.1416, found 359.1489.

(E)-1,3-二(3,4-二甲氧基苯基)丙-2-烯-1-酥(A9): 淡 黄色粉末, 产率 $57.52 \%$. m.p. 105.5 $107.2{ }^{\circ} \mathrm{C}$ (Lit. ${ }^{[13]}$ $\left.102 \sim 104{ }^{\circ} \mathrm{C}\right)$.

(E)-3-(3,4-二甲氧基苯基)-1-(4-甲氧基苯基)丙-2烯-1-酩(A10): 黄色粉末, 产率 18.27\%. m.p. 77.0 84.3 ${ }^{\circ} \mathrm{C}$ (Lit. $\left.{ }^{[14]} 90 \sim 92{ }^{\circ} \mathrm{C}\right) ;{ }^{1} \mathrm{H}$ NMR $\left(\mathrm{CDCl}_{3}\right) \delta: 8.040(\mathrm{~d}, J=$ $\left.8.4 \mathrm{~Hz}, 2 \mathrm{H}, \mathrm{H}-2^{\prime}, \mathrm{H}-6^{\prime}\right), 7.758$ (d, $\left.J=15.6 \mathrm{~Hz}, 1 \mathrm{H}, \mathrm{H}-\beta\right)$, 7.407 (d, $J=15.6 \mathrm{~Hz}, 1 \mathrm{H}, \mathrm{H}-\alpha), 7.235$ (dd, $J=1.8,8.4 \mathrm{~Hz}$, 1H, H-6), 7.229 (d, $J=1.2 \mathrm{~Hz}, 1 \mathrm{H}, \mathrm{H}-2$ ), 6.988 (d, $J=9.0$ Hz, 2H, H-3', H-5'), 6.917 (d, J=8.4 Hz, 1H, H-5), 3.958 $\left(\mathrm{s}, 3 \mathrm{H}, \mathrm{OCH}_{3}-3\right), 3.953\left(\mathrm{~s}, 3 \mathrm{H}, \mathrm{OCH}_{3}-4\right), 3.894(\mathrm{~s}, 3 \mathrm{H}$, $\left.\mathrm{OCH}_{3}-4^{\prime}\right)$; ESI-MS m/z: $299.2(\mathrm{M}+1)^{+}$.

(E)-3-(4-羟基-3-甲氧基苯基)-1-(2,4-二甲氧基苯基) 丙-2-烯-1-酮(A11): 黄色粉末, 产率 28.47\%. 137.1 139.3 (Lit. $\left.^{[15]} 92 \sim 94{ }^{\circ} \mathrm{C}\right) ;{ }^{1} \mathrm{H}$ NMR $\left(\mathrm{CDCl}_{3}\right) \delta: 7.713$ (d, $\left.J=8.4 \mathrm{~Hz}, 1 \mathrm{H}, \mathrm{H}-6^{\prime}\right), 7.591$ (d, $\left.J=15.6 \mathrm{~Hz}, 1 \mathrm{H}, \mathrm{H}-\beta\right)$, $7.328(\mathrm{~d}, J=15.6 \mathrm{~Hz}, 1 \mathrm{H}, \mathrm{H}-\alpha), 7.160(\mathrm{dd}, J=1.8,8.4 \mathrm{~Hz}$, 1H, H-6), 7.077 (d, $J=1.8 \mathrm{~Hz}, \mathrm{H}-2), 6.922$ (d, $J=8.4 \mathrm{~Hz}$, $1 \mathrm{H}, \mathrm{H}-5), 6.560$ (dd, $J=1.8,8.4 \mathrm{~Hz}, 1 \mathrm{H}, \mathrm{H}-5$ '), 6.500 (d, $\left.J=2.4 \mathrm{~Hz}, 1 \mathrm{H}, \mathrm{H}-3^{\prime}\right), 3.930$ (s, 3H, $\left.\mathrm{OCH}_{3}-2^{\prime}\right), 3.891$ (s, $\left.3 \mathrm{H}, \mathrm{OCH}_{3}-3\right), 3.856\left(\mathrm{~s}, 3 \mathrm{H}, \mathrm{OCH}_{3}-4\right.$ ) ; ESI-MS m/z: 315.2 $[\mathrm{M}+\mathrm{H}]^{+}$.
(E)-3-(3,4-二甲氧基苯基)-1-(3-羟基苯基)丙-2-烯1-酮(A12): 黄色粉末, 产率 17.2\%. m.p. 73.0 74.8 ${ }^{\circ} \mathrm{C}$ (Lit. $\left.{ }^{[16]} 115 \sim 116{ }^{\circ} \mathrm{C}\right) ;{ }^{1} \mathrm{H}$ NMR $\left(\mathrm{CDCl}_{3}\right) \delta: 7.776(\mathrm{~d}, J=$ $15.6 \mathrm{~Hz}, 1 \mathrm{H}, \mathrm{H}-\beta), 7.609$ (d, $\left.J=1.8 \mathrm{~Hz}, 1 \mathrm{H}, \mathrm{H}-2^{\prime}\right), 7.570$ (d, $J=7.8 \mathrm{~Hz}, 1 \mathrm{H}, \mathrm{H}-6 '), 7.377$ (t, $\left.J=7.8 \mathrm{~Hz}, 1 \mathrm{H}, \mathrm{H}-5^{\prime}\right)$, 7.369 (d, $J=15.6 \mathrm{~Hz}, 1 \mathrm{H}, \mathrm{H}-\alpha), 7.229$ (dd, $J=1.8,8.4 \mathrm{~Hz}$, 1H, H-6), 7.151 (d, $J=1.8 \mathrm{~Hz}, 1 \mathrm{H}, \mathrm{H}-2), 7.110$ (dd, $J=$ 2.4, $8.4 \mathrm{~Hz}, 1 \mathrm{H}, \mathrm{H}-4$ '), 6.894 (d, $J=8.4 \mathrm{~Hz}, 1 \mathrm{H}, \mathrm{H}-5$ ), $3.946\left(\mathrm{~s}, 3 \mathrm{H}, \mathrm{OCH}_{3}-3\right), 3.932\left(\mathrm{~s}, 3 \mathrm{H}, \mathrm{OCH}_{3}-4\right)$; ESI-MS $m / z: 285.1[\mathrm{M}+\mathrm{H}]^{+}$.

(E)-1-(4-差基苯基)-3-(4-甲氧基苯基)丙-2-烯-1-酮 (A13): 淡黄色粉末, 产率 93.64\%. m.p. 188.5 190.2 ${ }^{\circ} \mathrm{C}$ (Lit. ${ }^{[17]} 180 \sim 182{ }^{\circ} \mathrm{C}$ ).

(E)-1-(4-差基苯基)-3-(3,4,5-三甲氧基苯基)丙-2烯-1-酮(A14): 黄色粉末，产率 23.31\%. m.p. 230.2 235.8 ( Lit. $^{[18]} 236 \sim 238{ }^{\circ} \mathrm{C}$ ).

(E)-1,3-二(4-羟基苯基)丙-2-烯-1-酮(A15): 黄色粉 末, 产率 65.82\%. m.p. 200.4 202.2 ${ }^{\circ} \mathrm{C}$ (Lit. ${ }^{[19]}$ 196 198 $\left.{ }^{\circ} \mathrm{C}\right)$.

(E)-3-(4-差基苯基)-1-(2,4-二甲氧基苯基)丙-2-烯-1酮(A16): 黄色粉末, 产率 88.64\%. m.p. 128.2 130.9 ${ }^{\circ} \mathrm{C}$ (Lit. $\left.{ }^{[6]} 92 \sim 94{ }^{\circ} \mathrm{C}\right) ;{ }^{1} \mathrm{H}$ NMR $\left(\mathrm{CDCl}_{3}\right) \delta: 7.735$ (d, $J=9.0$ Hz, 1H, H-6'), 7.635 (d, $J=15.6$ Hz, 1H, H- $\beta$ ), 7.489 (d, $J=8.4 \mathrm{~Hz}, 2 \mathrm{H}, \mathrm{H}-2, \mathrm{H}-6), 7.373$ (d, $J=16.2 \mathrm{~Hz}, 1 \mathrm{H}, \mathrm{H}-\alpha$ ), $6.861(\mathrm{~d}, J=8.4 \mathrm{~Hz}, 2 \mathrm{H}, \mathrm{H}-5, \mathrm{H}-3), 6.560$ (dd, $J=2.4,8.4$ Hz, 1H, H-5'), 6.498 (d, J=1.8 Hz, 1H, H-3'); ESI-MS $m / z: 284.9[\mathrm{M}+\mathrm{H}]^{+}$.

(E)-1,3-二(3-羟基苯基)丙-2-烯-1-酮(A17)：黄色粉 末, 产率 13.7\%. m.p. 191 $193{ }^{\circ} \mathrm{C}$ (Lit. ${ }^{[20]} 155 \sim 156{ }^{\circ} \mathrm{C}$ ); ${ }^{1} \mathrm{H}$ NMR (DMSO- $d_{6}$ ) $\delta: 9.775$ (s, 1H, OH-3'), 9.640 (s, 1H, OH-3), 7.712 (d, $J=15.6 \mathrm{~Hz}, 1 \mathrm{H}, \mathrm{H}-\beta), 7.590$ (d, $J=16.2$ Hz, 1H, H- $\alpha$ ), 7.420 (s, 1H, H-2'), 7.351 (t, $J=8.4$ Hz, 1H, H-5'), 7.292 (d, J=7.2 Hz, 1H, H-6), 7.236 (t, $J=7.2 \mathrm{~Hz}$, 1H, H-5), 7.184 (s, 1H, H-2), 7.040 (d, J=7.2 Hz, 1H, H-4), 7.040 (d, $J=7.2 \mathrm{~Hz}, 1 \mathrm{H}, \mathrm{H}-4)$; ESI-MS $m / z$ : 240.9 $[\mathrm{M}+\mathrm{H}]^{+}$.

\section{2 .2 螺杂环 $\mathbf{B}$ 的合成}

反应采用一锅煮的方法: 在甲醇中加入等物质量的 A 类化合物、靛红和肌氨酸, 加热回流 $4 \sim 8$ h. 用 TLC 监测反应的进行, 反应完后, 反应液中加入 1 倍左右于 反应液体积的水, 析出沉淀, 真空干燥. B1 用丙醖: 水 $(V: V=2: 1)$ 溶液重结晶纯化. 用硅胶柱层析纯化 B2、

B3 和 B4, 洗脱溶剂均为石油醚: 乙酸乙酯(体积比约 1: 1 左右 $)$.

4'-(3,4-二羟基苯基)-3'-[(2,4-二甲氧基苯基)碳酰 
基]-1'-甲基-螺[2,3"]羊弪吲哚-吡咯烷(B1): 淡黄色粉末, 产率 30.63\%. m.p. 137 $140.5{ }^{\circ} \mathrm{C} ;{ }^{1} \mathrm{H}$ NMR (DMSO- $d_{6}$ ) $\delta$ : $7.558\left(\mathrm{~d}, J=9.0 \mathrm{~Hz}, 1 \mathrm{H}, \mathrm{Ar}-\mathrm{H}^{2 "}\right), 7.147$ (dd, $J=2.4,9.0$ $\left.\mathrm{Hz}, 1 \mathrm{H}, \mathrm{Ar}-\mathrm{H}^{5 "}\right), 6.965 \sim 7.069$ (m, 2H, Ar- $\left.\mathrm{H}^{3 "}, \mathrm{Ar}-\mathrm{H}^{4 "}\right)$, $6.848\left(\mathrm{~d}, J=9.0 \mathrm{~Hz}, 1 \mathrm{H}, \mathrm{Ar}-\mathrm{H}^{6}\right), 6.661(\mathrm{~d}, J=1.8 \mathrm{~Hz}, 1 \mathrm{H}$, $\left.\mathrm{Ar}-\mathrm{H}^{2}\right), 6.612\left(\mathrm{dd}, J=1.8,8.4 \mathrm{~Hz}, 1 \mathrm{H}, \mathrm{Ar}-\mathrm{H}^{6}\right), 6.592$ (s, $\left.1 \mathrm{H}, \operatorname{Ar}-\mathrm{H}^{5^{\prime}}\right), 6.581$ (d, J=1.8 Hz, 1H, Ar-H $\left.{ }^{3}\right), 6.558$ (d, $J=7.8 \mathrm{~Hz}, 1 \mathrm{H}, \mathrm{Ar}-\mathrm{H}^{5}$ ), 4.479 (d, $\left.J=9.6 \mathrm{~Hz}, 1 \mathrm{H}, 3-\mathrm{CH}\right)$, $4.096 \sim 4.141(\mathrm{~m}, 1 \mathrm{H}, 4-\mathrm{CH}), 3.685\left(\mathrm{~s}, 3 \mathrm{H}, 2{ }^{\prime}-\mathrm{OCH}_{3}\right)$, $3.618\left(\mathrm{~s}, 3 \mathrm{H}, 4{ }^{\prime}-\mathrm{OCH}_{3}\right), 3.140 \sim 3.166\left(\mathrm{~m}, 1 \mathrm{H}, 5-\mathrm{CH}_{2}\right)$, 1.966 (s, 3H, $\left.\mathrm{NCH}_{3}\right)$. HRMS calcd for $\mathrm{C}_{27} \mathrm{H}_{26} \mathrm{~N}_{2} \mathrm{O}_{6}[\mathrm{M}+$ $\mathrm{H}]^{+}$475.1791, found 475.1865.

4'-(3,4-二羟基苯基)-3'-[(2-氯苯基)碳酰基]-1'-甲基螺[2,3"]差弪吲哚一吡咯烷(B2): 棕黄色粉末, 产率 $60.16 \%$. m.p. 188.4 189.55 ${ }^{\circ} \mathrm{C} ;{ }^{1} \mathrm{H}$ NMR $\left(\mathrm{CDCl}_{3}\right), \delta$ : $9.321(\mathrm{~s}, 1 \mathrm{H}$, N-H), 7.275 (d, J=7.8 Hz, 1H, Ar-H' $\left.{ }^{6}\right), 7.184 \sim 7.224(\mathrm{~m}$, $\left.4 \mathrm{H}, \mathrm{Ar}-\mathrm{H}^{3^{\prime}}, \mathrm{Ar}-\mathrm{H}^{4^{\prime}}, \mathrm{Ar}-\mathrm{H}^{5^{\prime}}, \mathrm{Ar}-\mathrm{H}^{2 "}\right), 7.017 \sim 7.050$ (m, 4H, $\left.\mathrm{Ar}-\mathrm{H}^{2}, \mathrm{Ar}-\mathrm{H}^{3 "}, \mathrm{Ar}-\mathrm{H}^{4 "}, \mathrm{Ar}-\mathrm{H}^{5 "}\right), 6.832$ (d, J=7.2 Hz, 1H, $\left.\operatorname{Ar}-\mathrm{H}^{6}\right), 6.766\left(\mathrm{~d}, J=7.2 \mathrm{~Hz}, 1 \mathrm{H}, \mathrm{Ar}-\mathrm{H}^{5}\right), 4.633$ (t, $J=9.0$ $\mathrm{Hz}, 1 \mathrm{H}, 3-\mathrm{CH}), 4.214$ (q, $J=9.0 \mathrm{~Hz}, 16.8 \mathrm{~Hz}, 1 \mathrm{H}, 4-\mathrm{CH})$, $3.434\left(\mathrm{t}, J=9.0 \mathrm{~Hz}, 1 \mathrm{H}, 5-\mathrm{CH}_{2}\right), 3.337 \sim 3.373(\mathrm{~m}, 1 \mathrm{H}$, $\left.5-\mathrm{CH}_{2}\right), 3.485 \sim 3.501\left(\mathrm{~m}, 1 \mathrm{H}, 5-\mathrm{CH}_{2}\right), 2.062(\mathrm{~s}, 3 \mathrm{H}$, 1-N-CH 3 ). HRMS calcd for $\mathrm{C}_{25} \mathrm{H}_{21} \mathrm{ClN}_{2} \mathrm{O}_{4}[\mathrm{M}+\mathrm{H}]^{+}$ 449.1190, found 449.1252.

4'-(3,4-二甲氧基苯基)-3'-[(2-氯苯基)碳酰基]-1'-甲 基一螺 [2,3"]羊弪吲哚一吡咯烷(B3)：淡黄色粉末，产率 38.07\%. m.p. $123.5 \sim 128.9{ }^{\circ} \mathrm{C} ;{ }^{1} \mathrm{H}$ NMR $\left(\mathrm{CDCl}_{3}\right) \delta: 7.460$ $\left(\mathrm{d}, J=7.8 \mathrm{~Hz}, 1 \mathrm{H}, \mathrm{Ar}-\mathrm{H}^{6}\right), 7.351 \sim 7.394\left(\mathrm{~m}, 3 \mathrm{H}, \mathrm{Ar}-\mathrm{H}^{3^{\prime}}\right.$, $\left.\mathrm{Ar}-\mathrm{H}^{4^{\prime}}, \mathrm{Ar}-\mathrm{H}^{2 "}\right), 7.088 \sim 7.187\left(\mathrm{~m}, 5 \mathrm{H}, \mathrm{Ar}-\mathrm{H}^{2}, \mathrm{Ar}-\mathrm{H}^{5^{\prime}}\right.$, $\left.\mathrm{Ar}-\mathrm{H}^{3 "}, \mathrm{Ar}-\mathrm{H}^{4 "}, \mathrm{Ar}-\mathrm{H}^{5 "}\right), 6.877\left(\mathrm{~d}, J=7.8 \mathrm{~Hz}, 1 \mathrm{H}, \mathrm{Ar}-\mathrm{H}^{6}\right)$, $6.830\left(\mathrm{~d}, J=7.8 \mathrm{~Hz}, 1 \mathrm{H}, \mathrm{Ar}-\mathrm{H}^{5}\right), 4.676(\mathrm{t}, J=9.0 \mathrm{~Hz}, 1 \mathrm{H}$, $3-\mathrm{CH}), 4.405 \sim 4.431(\mathrm{~m}, 1 \mathrm{H}, 4-\mathrm{CH}), 3.917$ (s, 6H, $\left.\mathrm{Ar}-3-\mathrm{OCH}_{3}, \mathrm{Ar}-4-\mathrm{OCH}_{3}\right), 3.716 \sim 3.735\left(\mathrm{~m}, 1 \mathrm{H}, 5-\mathrm{CH}_{2}\right)$, $3.485 \sim 3.501\left(\mathrm{~m}, 1 \mathrm{H}, 5-\mathrm{CH}_{2}\right), 2.175\left(\mathrm{~s}, 3 \mathrm{H}, 1-\mathrm{N}-\mathrm{CH}_{3}\right)$; ESI-MS $m / z \quad 477.0[\mathrm{M}+\mathrm{H}]^{+}$; HRMS calcd for $\mathrm{C}_{27} \mathrm{H}_{25} \mathrm{ClN}_{2} \mathrm{O}_{4}[\mathrm{M}+\mathrm{H}]^{+}$477.1503, found 477.1590.

4'-(3,4-二羟基苯基)-3'-[(4-乙氧基苯基)碳酰基]-1'甲基-螺[2,3"]羟吲哚一吡咯烷(B4)：黄色粉末，产率 25.3\%. m.p. $112.1 \sim 115.3{ }^{\circ} \mathrm{C} ;{ }^{1} \mathrm{H}$ NMR $\left(\mathrm{CDCl}_{3}\right) \delta: 7.334$ (d, $\left.J=9.0 \mathrm{~Hz}, 2 \mathrm{H}, \mathrm{Ar}-\mathrm{H}^{2}, A r-\mathrm{H}^{6}\right), 7.203$ (d, $J=1.8 \mathrm{~Hz}$, $\left.1 \mathrm{H}, \operatorname{Ar}-\mathrm{H}^{2}\right), 7.156$ (d, J=7.2 Hz, 1H, Ar-H" $\left.{ }^{5 "}\right), 6.995 \sim$ $7.018\left(\mathrm{~m}, 1 \mathrm{H}, \mathrm{Ar}-\mathrm{H}^{3 "}\right), 6.910 \sim 6.935\left(\mathrm{~m}, 1 \mathrm{H}, \mathrm{Ar}-\mathrm{H}^{4 "}\right)$, $6.889\left(\mathrm{dd}, J=1.8,8.4 \mathrm{~Hz}, 1 \mathrm{H}, \operatorname{Ar}-\mathrm{H}^{6}\right), 6.799$ (d, $J=7.8$ $\left.\mathrm{Hz}, 1 \mathrm{H}, \mathrm{Ar}-\mathrm{H}^{2 "}\right), 6.519$ (d, J=7.2 Hz, 1H, Ar- $\left.\mathrm{H}^{5}\right), 6.483$ (d, $\left.J=9.0 \mathrm{~Hz}, 2 \mathrm{H}, \mathrm{Ar}-\mathrm{H}^{3^{\prime}}, \mathrm{Ar}-\mathrm{H}^{5^{\prime}}\right), 4.424 \sim 4.476(\mathrm{~m}, 2 \mathrm{H}$,
5- $\mathrm{CH}_{2}$ ), 3.875 (q, $J=7.2 \mathrm{~Hz}, 2 \mathrm{H}, 4$ '- $\left.-\mathrm{OCH}_{2}\right), 3.572$ (t, $J=$ $9.0 \mathrm{~Hz}, 1 \mathrm{H}, 3-\mathrm{CH}), 3.404 \sim 3.431(\mathrm{~m}, 1 \mathrm{H}, 4-\mathrm{CH}), 2.106(\mathrm{~s}$, $\left.3 \mathrm{H}, \mathrm{N}-\mathrm{CH}_{3}\right), 1.312\left(\mathrm{t}, J=7.2 \mathrm{~Hz}, 3 \mathrm{H}, 4{ }^{\prime}-\mathrm{OCH}_{2} \mathrm{CH}_{3}\right)$; ESI-MS $m / z$ : 458.9; HRMS calcd for $\mathrm{C}_{27} \mathrm{H}_{26} \mathrm{~N}_{2} \mathrm{O}_{5}[\mathrm{M}+$ $\mathrm{H}]^{+}$459.1842, found 459.1937 .

\section{3 晶体培养与单晶衍射}

取少量 $\mathbf{B} 1$ 用丙酮 : 水 $(V: V=2: 1)$ 的混合溶液溶 解, 置于 $4{ }^{\circ} \mathrm{C}$ 环境下溶剂缓慢挥发, $6 \mathrm{~d}$ 之后得到黄色柱 状晶体. 将 B1 的晶体在 $293 \mathrm{~K}$ 下用 Bruker SMARTAPEXII-CCD X 射线单晶衍射仪进行结构测定. 采用 $\mathrm{Mo} \mathrm{K} \alpha$ 射线 $(\lambda=0.71073 \AA)$, 石墨晶体单色器, 在 $293 \mathrm{~K}$ 下，以 $\rho-\omega$ 扫描方式在 $2.3^{\circ}<\theta<28.1^{\circ}$ 收集衍射数据 (SMART 程序).

\section{4 体外抗氧化活性: 对 DPPH 的抑制率}

用无水乙醇溶解 $\mathrm{DPPH}$, 配置母液 $\left(7.5 \times 10^{4}\right.$ $\mu \mathrm{mol} / \mathrm{L}), 0 \sim 4{ }^{\circ} \mathrm{C}$ 下避光保存. 使用前用无水乙醇稀释 到 $150 \mu \mathrm{mol} / \mathrm{L}$. 用 DMSO 溶解化合物, 其初始浓度 $1.0 \times 10^{4} \mathrm{mg} / \mathrm{L}, 0 \sim 4{ }^{\circ} \mathrm{C}$ 下避光保存. 使用前用无水乙醇 稀释到 $20 \mathrm{mg} / \mathrm{L}$. 在 96 孔板中加入 $120 \mu \mathrm{L} \mathrm{DPPH}$ 溶液 和 $80 \mu \mathrm{L}$ 化合物溶液, 使总体积为 $200 \mu \mathrm{L}$, 混匀, 于室 温 $\left(25{ }^{\circ} \mathrm{C}\right)$ 下放置 $30 \mathrm{~min}$, 记为溶液 $\mathrm{i}$; 在 96 孔板中加入 $80 \mu \mathrm{L}$ 化合物溶液和 $120 \mu \mathrm{L}$ 无水乙醇, 使总体积为 200 $\mu \mathrm{L}$, 混匀, 于室温 $\left(25^{\circ} \mathrm{C}\right)$ 下放置 $30 \mathrm{~min}$, 记为溶液 $\mathrm{j}$; 在 96 孔板中加入 $120 \mu \mathrm{L} \mathrm{DPPH}$ 溶液和 $80 \mu \mathrm{L}$ 无水乙醇, 使 总体积为 $200 \mu \mathrm{L}$, 混匀, 于室温 $\left(25^{\circ} \mathrm{C}\right)$ 下放置 $30 \mathrm{~min}$, 记为溶液 $\mathrm{c}$; 在酶标仪上 $517 \mathrm{~nm}$ 测定其吸光度分别为 $A_{\mathrm{i}}, A_{\mathrm{j}}, A_{\mathrm{c}}$. 根据公式：抑制率 $(\%)=\left[1-\left(A_{\mathrm{i}}-A_{\mathrm{j}}\right) /\right.$ $\left.A_{\mathrm{c}}\right] \times 100 \%$ ，计算待测液对 $\mathrm{DPPH}$ 的抑制率. 测试时以 䚞皮素和维生素 C (Vc) 做对照. 每个化合物每次测定 3 个复孔，测定 3 次重复实验.

\section{References}

[1] Cioffi, G.; Morales Escobar, L.; Braca, A.; De Tommasi, N. J. Nat. Prod. 2003, 66, 1061.

[2] Wu, J. Z.; Li, J. L.; Cai, Y. P.; Pan, Y.; Ye, F. Q.; Zhang, Y. L.; Zhao, Y. J.; Yang, S. L.; Li, X. K.; Liang, G. J. Med. Chem. 2011, 54: 8110.

[3] Martens, S.; Mithofer, A. Phytochemistry 2005, 66, 2399.

[4] Chen, Z. H.; Zheng, C. J.; Sun, L. P.; Piao, H. R. Eur. J. Med. Chem. 2010, 45, 5739.

[5] Dominguez, J. N.; Leon, C.; Rodrigues, J.; Gamboa de Dominguez, N.; Gut, J.; Rosenthal, P. J. J. Med. Chem. 2005, 48, 3654.

[6] Guantai, E. M.; Ncokazi, K.; Egan, T. J.; Gut, J.; Rosenthal, P. J.; Bhampidipati, R.; Kopinathan, A.; Smith, P. J.; Chibale, K. J. Med. Chem. 2011, 54, 3637.

[7] Ghosh, A.; Mandal, S.; Banerji, A.; Kar, M.; Banerji, J. Nat. Prod. Commun. 2009, 4, 209.

[8] Kozlowski, D.; Trouillas, P.; Calliste, C.; Marsal, P.; Lazzaroni, R.; Duroux, J. L. J. Phys. Chem. A 2007, 111, 1138. 
[9] Padhye, S.; Ahmad, A.; Oswal, N.; Sarkar, F. H. J. Hematol. Oncol. 2009, 2, 38.

[10] Kumar, R. R.; Perumal, S.; Senthilkumar, P.; Yogeeswari, P.; Sriram, D. J. Med. Chem. 2008, 51, 5731.

[11] Sogawa, S.; Nihro, Y.; Ueda, H.; Izumi, A.; Miki, T.; Matsumoto, H.; Satoh, T. J. Med. Chem. 1993, 36, 3904.

[12] Iwata, S.; Nishino, T.; Nagata, N.; Satomi, Y.; Nishino, H.; Shibata, S. Biol. Pharm. Bull. 1995, 18, 1710.

[13] Lopez, S. N.; Castelli, M. V.; Zacchino, S. A.; Dominguez, J. N.; Lobo, G.; Charris-Charris, J.; Cortes, J. C.; Ribas, J. C.; Devia, C.; Rodriguez, A. M.; Enriz, R. D. Bioorg. Med. Chem. 2001, 9, 1999.

[14] Bag, S.; Ramar, S.; Degani, M. S. Med. Chem. Res. 2009, 18, 309.

[15] Guantai, E. M.; Ncokazi, K.; Egan, T. J.; Gut, J.; Rosenthal, P. J.; Bhampidipati, R.; Kopinathan, A.; Smith, P. J.; Chibale, K. J. Med.
Chem. 2011, 54, 3637.

[16] Hsieh, H. K.; Lee, T. H.; Wang, J. P.; Wang, J. J.; Lin, C. N. Pharm. Res. 1998, 15, 39.

[17] Gul, H. I.; Yerdelen, K. O.; Das, U.; Gul, M.; Pandit, B.; Li, P. K.; Dimmock, J. R. Chem. Pharm. Bull. (Tokyo) 2008, 56, 1675.

[18] Acharya, B. N.; Saraswat, D.; Tiwari, M.; Shrivastava, A. K.; Ghorpade, R.; Bapna, S.; Kaushik, M. P. Eur. J. Med. Chem. 2010, 45,430 .

[19] Zhang, X. W.; Zhao, D. H.; Quan, Y. C.; Sun, L. P.; Yin, X. M.; Guan., L. P. Med. Chem. Res. 2010, 19, 403.

[20] Viqar, U. A.; Mohammad, G. S.; Mushtaq, N.; Mohammad, F. V. J. Nat. Prod. 1992, 55, 956.

[21] Kumar, R. R.; Perumal, S.; Senthilkumar, P.; Yogeeswari, P.; Sriram, D. Tetrahedron 2008, 64, 2962. 